\title{
An Experiment in Design and Analysis of Real-Time Applications
}

\author{
Roman Gumzej*, Domen Verber*, Matjaž Colnarič*, \\ Jean-Philippe Babau ${ }^{+}$, Jacques J. Skubich ${ }^{+}$ \\ *University of Maribor, Faculty of Electrical Engineering and Computer science, Slovenia \\ + INSA de Lyon, France
}

In the paper some experiences of joining two methodologies, which were originally independently developed in different institutions, with the goal to overcome the possible discrepancies due to the separate design of the hardware and the software part of an embedded real-time application are presented.

Based on Multiprocessor PEARL, Specification PEARL has been developed in FERI, Maribor. Hardware and system architecture of an application can be described and gradually refined. Application software can be designed using LACATRE tool, developed at INSA, Lyon. Decisions about the application design taken in each tool have influence to the ones taken in the other, thus allowing for parallel design of both parts.

Both designs are subsequently verified and eventually joined for feasibility estimation by co-simulation. The application program is coded using the ObjectPEARL language. The real-time system design cycle is closed by the execution time analysis and measurements upon which it is then considered about further program and/or hardware part reconfiguration. This feature is supported by the specific compiler, which includes the execution time analyser.

The article reports on the work that was done in the framework of the PROTEUS project in co-operation of the teams from FERI Maribor, Slovenia, and INSA de Lyon, France.

\section{Introduction}

Real-time systems have traditionally been built using specialized concepts and tools. They have been handcrafted and tuned through many iterations of different tests. Their dependability has most often been assured by multiple redundancy and over-scaled components. In order to change this, different modeling techniques have been used. Only a few of them reached a de facto standardization level, and even those were sometimes outvoted by practitioners with addictions to specific tools and/or $p$ ages which "served the purpose".

Real-time systems are expected to conform to safety restrictions beside the real-time imposed limitations. It is necessary to design them in a way that will enable early reasoning about their structure and timing as well as effective verification of their correctness.

A number of formal descriptions have been devised to cope with this feature: differential equations supported formalisms which describe the systems' functional and temporal behaviour [10], formal languages and timed automata [1, 6], combinations of conventional CASE methods and state charts have been used [34]. Graphical techniques with the same expressing power as their formal language counterparts have been defined [9]. While enabling formal verification, most of these methods lack the versatility of basic constructs and user friendliness. Therefore, graphical formalisms with a larger set of basic constructs have been defined (e.g.: CSR/CCSR [19], TTM/RTTL [28], LACATRE [31]), while keeping enough strictness to enable verification.

The developers of CASE methods and tools have also identified this gap in embedded systems design and are developing methods for unified representations of common purpose and 
embedded systems design issues (e.g.: HRTHOOD [5], ObjecTime [27], Shlaer-Mellor [33], LIMITS [26], UML $[3,35])$. The possibility of an error in the specifications or design is being reduced and introducing controlled changes is made easier and less error prone. This should also supplement higher quality of the resulting systems.

Research in hardware and software co-design deals with the optimization of the processor load by distributing it among hardware and software or the minimization of hardware cost. The most useful advantage is that the designer has the ability to easily and quickly trade-off hardware against software realisations for individual function blocks. Usually a known hardware specification language (e.g.: VHDL, HDL) is combined with a high-level programming language (e.g.: DFL, C) for multiprocessor programming (e.g.: VULCAN [14], COWARE [23]). Some used a unified programming language to specify behaviour and structure, describing processors, peripheral devices and communication interfaces (e.g.: CHINOOK [4]). Dedicated state transition automata [32] are often used as the basic computation model (e.g.: POLIS [2]). Co-designing systems with time limitations led to the introduction of scheduling algorithms into co-design and simulation (e.g. [24]).

The research presented in this paper is dealing with the domain of generic computer control systems. Usually, there is not much room for the real HW-SW co-design: most of hardware solutions are given and the only choice the designer has is to select the most appropriate ones and design the application based on those. Thus, the main contribution we see in

- the possibility of designing the HW and the SW part in parallel, thus allowing early interference and adaptations on both sides to suit the needs and possibilities of the other, and

- the possibility of validating the functional and the temporal properties of the design in a holistic way, considering properties of both parts and in all stages of the design.

Because of the fact that already existing and very diverse tools were taken for different tasks in real-time application design, it is obvious that that introduced certain limitations. In the conclusion the main discrepancies are listed together with the suggestions for their solution, which will be considered in the next versions of the tools.

Further, at this stage only a part of the application design process was covered by the proposed approach. Although necessary and challenging, the early phase of requirements analysis has not been covered yet.

In the paper, first the overall concept of the merged methodology will be outlined. Then, the tools will be presented separately and finally, the suggestions for changes to allow for more successful integration will be enumerated.

\section{Merging the Approaches}

Development cycle of the application as proposed in this paper is shown in Figure 1.

As already mentioned, for the time being, early stages of the development cycle like requirements analysis are not supported. Also, to come to the first outline of the top-level overall design, some preliminary decisions must be made, which can be re-considered and certainly refined during the design process.

The design can then start in parallel on both software and hardware architecture levels. Using Specification PEARL (SPEARL) [7, 11] basic description of hardware and system architecture is elaborated. The designer first sets up the coarse structure of the system as well as implementation parameters, which are then gradually detailed with time. When at least the outline of the hardware architecture is set up, software units (collections) may be associated with it. To allow for that, during the development this model is detailed by information from LACATRE (e.g. number and basic structure of tasks and other objects). Specification PEARL enables early reasoning about the system integration, but at the same time the hierarchical structure of the corresponding design tool also enables top-down stepwise refinement.

At the same time, program specific model of the application can already be developed by LACATRE. The application is first represented by abstract elements such as tasks, semaphores etc. During the development process these elements are further refined with additional details and 
attributes. The LACATRE tool also performs structure and behaviour verification of the program model by checking whether the tasks are well formed and trying to avoid possible deadlocks and bottlenecks. In the early phases, probably a lot of detailed algorithms will be replaced by placeholders and will be gradually more and more detailed during the forthcoming iterations.

During that time, both processes can have impact on the overall design, thus allowing for optimal implementation. Direct interference between the LACATRE and SPEARL, however, is not possible in this implementation, and will be subject of the forthcoming versions of the tools.

When the design on a certain level is complete, the system and the application descriptions are merged. As programs in standard PEARL consist of system and problem parts, the problem part of the PEARL program is a result of LACATRE and the system part is produced by SPEARL. The bold arrows represent that this activity is automated while, e.g., the evaluation is done manually.

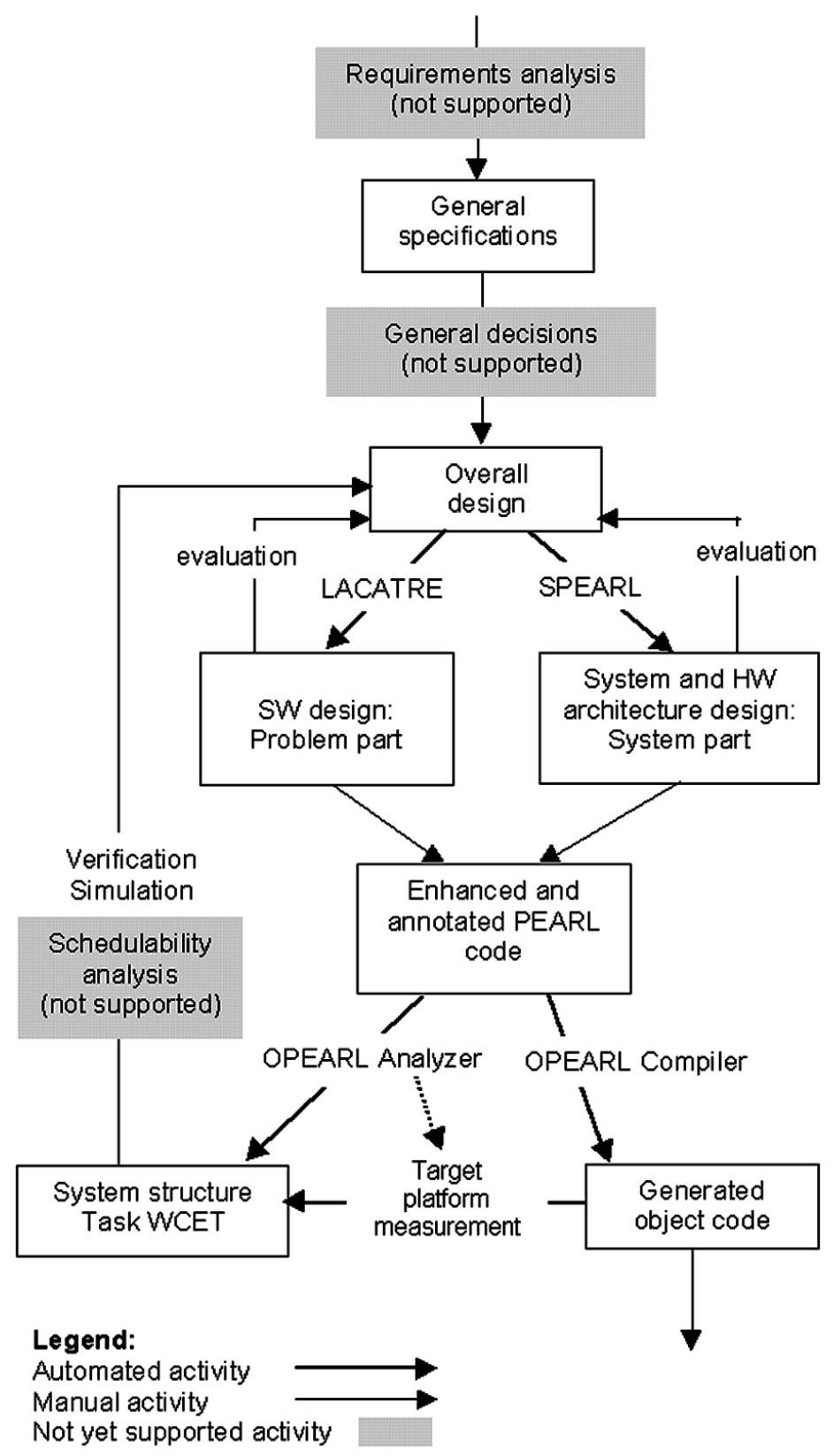

Fig. 1. Development Cycle of the Application. 
The PEARL code is then input into the ObjectPEARL (OPEARL) compiler with a builtin execution time analyser. In early phases only coarse design analysis is done in order to obtain a rough estimation of system's structure and behaviour. During the design process, the estimation becomes gradually more precise and reliable until finally the object code for the target platform can be generated.

At that stage already a good estimation of task's execution times can be done, thus providing reliable information for verification of system's temporal behaviour. The pessimism necessarily involved is minimized by automated measurement of the pilot code generated by the analyser and supported by the background debugging mode facility of the employed microprocessor.

Better option at this stage would be to perform a thorough schedulability analysis $[18]$ what we find feasible, but is for the time being not supported.

The estimated or measured temporal behaviour is checked against the specifications. It can also be simulated using the architectural model resulting from the system part, and the tasks' behaviour described in the problem part. The cycle closes by returning to the overall design, which can be changed or refined according to the results of the validation.

\section{Specification PEARL}

Specification PEARL is a hardware/software architecture specification and description language. It originates from PEARL for distributed systems [22]. The latter was defined to support the design of real-time systems and applications, which often are of the distributed nature. They represent an extension to the Full PEARL [21] standards' SYSTEM part, which describes the hardware units being addressed in the PROBLEM part of the PEARL program. The description of the hardware architecture consists of the top-level "station layer" and the detailed-level "component layer". There is a separate "program layer", which depicts the software architecture.

Specification PEARL extends the standard PEARL for distributed systems in the manner consistently with the standard. Some constructs have been added in order to enable the description of asymmetrical distributed multiprocessor systems and the peripherals, attached to the system. The parameters of the Specification PEARL components have been chosen based on the PEARL for distributed systems standard and our experience in designing an asymmetrical multiprocessor hardware platform [8] and analysing the worst case execution times of PEARL programs. However, although motivated by the particular architecture, the method is universal enough to model a wide variety of different embedded systems.

Specification PEARL has two representations: the graphical and the textual one. They have an equivalent translation from the graphical to the textual form and vice versa. Both representations are described in the sequel. The graphical symbols used to represent the components of Specification PEARL have been chosen in compliance with the philosophy of the LACATRE graphical constructs:

- a common symbol for every component group,

- easy recognition and differentiation between different components,

- specialised components are added additional graphical details in order to differentiate them from the other components in the group.

\subsection{The Textual Specification PEARL}

The textual system architecture description [12] consists of divisions, which describe the components of the system architecture and their interconnections by looking at those from different perspectives.

\section{Station Division}

In the station division, the processing nodes of the system are introduced, stating their most important characteristics. Stations are treated as black boxes with connectors, called "PORTS", by the other layers of the architecture description. PORTs are a means of communication between the stations of a system and are referenced by their program collection's tasks. A similar concept is also used in UML [20,30].

Typically, there are more stations in a system, so each of them is uniquely identified. Each 
station is associated with the state information, upon which it is being decided which application program collection is loaded and executed by the station.

Several types of stations have been defined. The default type is the BASIC station, which represents a general purpose processing node. To be able to describe asymmetrical architectures, two additional types of processing nodes have been defined: TASKPROC for task processors and KERNELPROC for operating system kernel processors [8]. This comes from a widely accepted concept of migrating the operating system functions to a separate processor, which enables better predictability of the actual program execution times.

To allow for the design of multiprocessor nodes, a "compound station" has been defined to be a set of stations, which are logically and/or physically strongly connected (they share the same connections with other stations or peripheral devices). A multiprocessor node is introduced by the PART OF attributes of the constituent processing nodes.

Since in embedded systems design (intelligent) peripheral devices are very important the PERIPHERAL station type was defined. The attributes of peripheral stations differ from other stations' attributes because of their large diversity. Hence, only a common denominator of their parameters is used. Their connections to the devices in the system are described by the attributes of their interface and PORTs (e.g.: the direction of data flow, the protocol used and any additional signals which may be necessary for the communication). To support schedulability analysis, every signal can be specified by its minimum inter-arrival time.

The basic components of a station are its processing elements, working stores and devices $(\mathrm{I} / \mathrm{O}$, timers, etc $)$. They are assigned parameters, which are important for the execution time analysis during application program compilation and the parameterisation of the RTOS and configuration manager.

\section{Net Division}

The topology of the system is described by portto-port connections. In net division the physical port-to-port connections between the stations of the system are listed giving their logical names and directions.

\section{System Division}

It represents an extended SYSTEM part, as obligatory in standard PEARL programs, which represents the programmer's view of the system. System division encapsulates the hardware description and the assignment of symbolic names to hardware devices. The described components from the station and net divisions are used.

\section{Configuration Division}

Configuration division describes the software architecture. The largest program component that is associated with a station and its state is a COLLECTION of MODULES. It is possible to specify under which conditions certain collections are removed from a station and which collections are loaded instead. These conditions are station state dependent.

Modules consist of TASKs, which may communicate through PORTs. Tasks are described by their trigger conditions and response times. Alternative implementations of tasks, which serve the purpose of increasing fault-tolerance and aid the feasibility of task scheduling, may be defined (during scheduling a task with shorter run time or longer response time can be scheduled in order to maintain the feasibility of the schedule). The port-to-port connections between tasks are described by their directions and line attributes. Line attributes state which connections are always followed and which can be chosen from a preference list.

\subsection{The Graphical Specification PEARL}

The graphical equivalent [11] of the textual Specification PEARL language has been devised in order to ease the exchange of ideas and graphical design of the specifications.

There are three layers in the graphical representation of the system architecture with the graphical constructs, which depict the same components, as described in the textual sections and which are given their properties as parameters: 


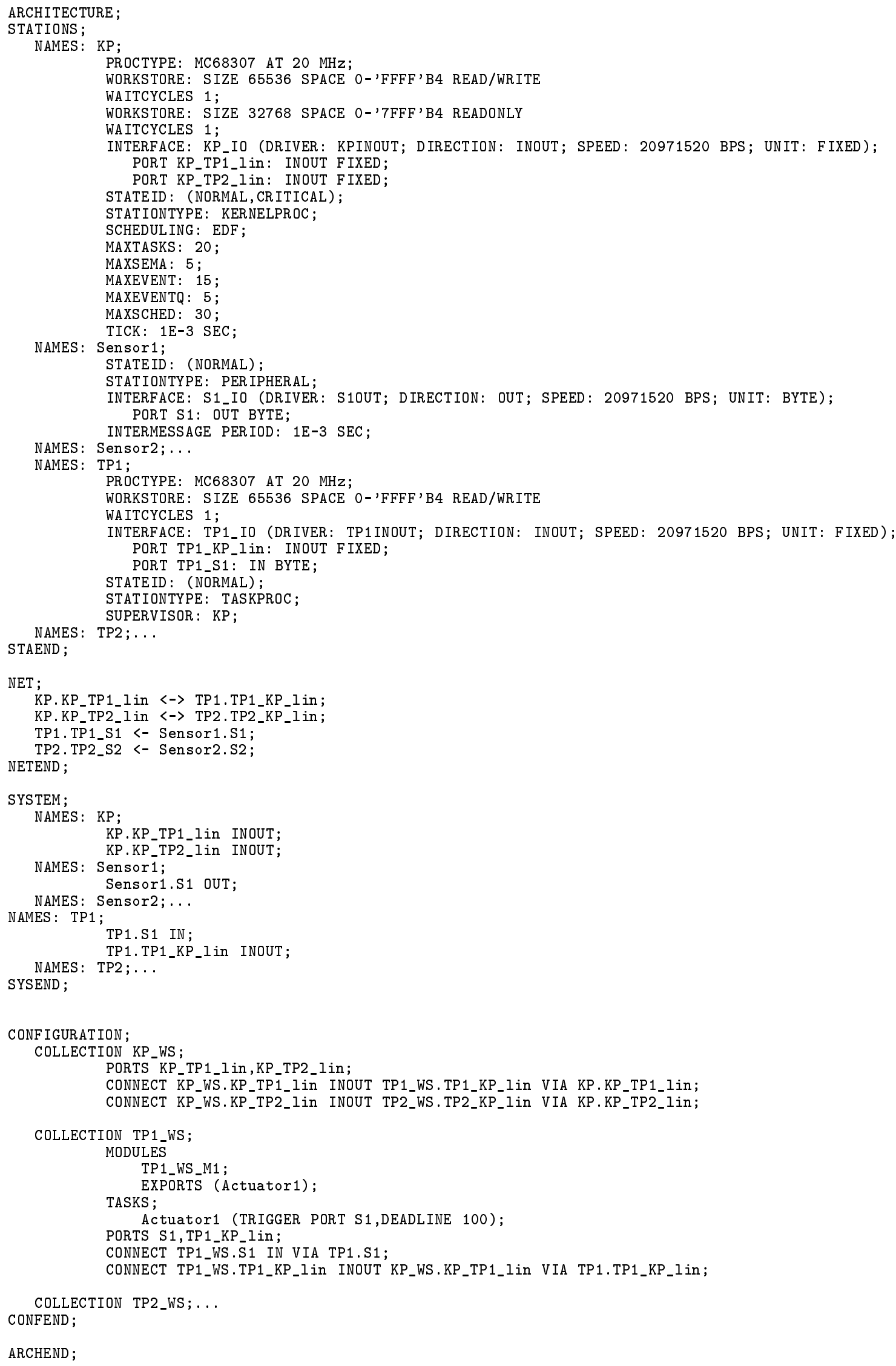

Fig. 2. Example Description of an Asymmetrical Control System. 
1. Station layer: STATIONS and PERIPHERALs with their interconnections,

2. Component layer: PROCTYPES, WORKSTORES and DEVICES of a STATION with their internal and global interconnections, and

3. Program layer: COLLECTIONs of MODULEs and TASKs, which are associated with the stations from the station layer.

\subsection{Hardware Configuration Verification}

The purpose of the hardware configuration verification is to ensure a complete and coherent hardware architecture model with enough timing information to enable integration verification with the program model.

There are three phases in the system model coherence checking:

1. Completeness check: "Are all stations in the hardware architecture fully described?" is the main question to be answered here. It is not possible to reason about the system feasibility without these hardware architecture properties. It is also important to check that every component, which is referenced in the configuration, actually exists with its description.

2. Range and compatibility check: it is always possible to make a mistake while inputting the values for the parameters. While some can only be detected by reviewing the design by the designer, some mistakes like range errors, where the input data differs in the order of magnitude from the expected value range, can be detected automatically. It is also possible that we connect two stations through interface with different properties, which will surely result in a communication malfunction in case the configuration doesn't change.

3. Software to hardware mapping check is the preparation to the integration verification and is described in the corresponding section.

\subsection{Example Description of an Asymmetrical Architecture}

To illustrate the expressive power and modelling capabilities of the Specification PEARL, the following example (Figure 2) is presented here. An asymmetrical architecture is considered. It consists of two embedded task processors TP1 and TP2, which are supervised by a common kernel processor KP. They are receiving input from two peripheral devices Sensorl and Sensor2 respectively. Their Actuator tasks are triggered by the signal from one or the other sensor. They are synchronised by the operating system being run by the KP station.

\section{Software Design}

\subsection{LACATRE}

LACATRE [31] is a language with graphical and textual modes; it has been conceived as a contribution to the development of design tools for applications relying on multitasking. It covers the end of preliminary design and detailed design of the software lifecycle and enhances the expression of the dynamic decomposition and task relationship. An application designed by means of graphical components of the LACATRE language is a 2-dimensional diagram made of LACATRE graphical objects (those commonly involved in most of real-time executives) and LACATRE connections (System calls) which appear as graphical links between the objects.

The language's textual form allows for the production of a textual LACATRE program associated to the graphical design. This textual form is especially interesting for the detailed design step because it permits an automatic code generation thanks to an ad hoc compiler, which means implementation of the application program in the chosen target system and operating system. 


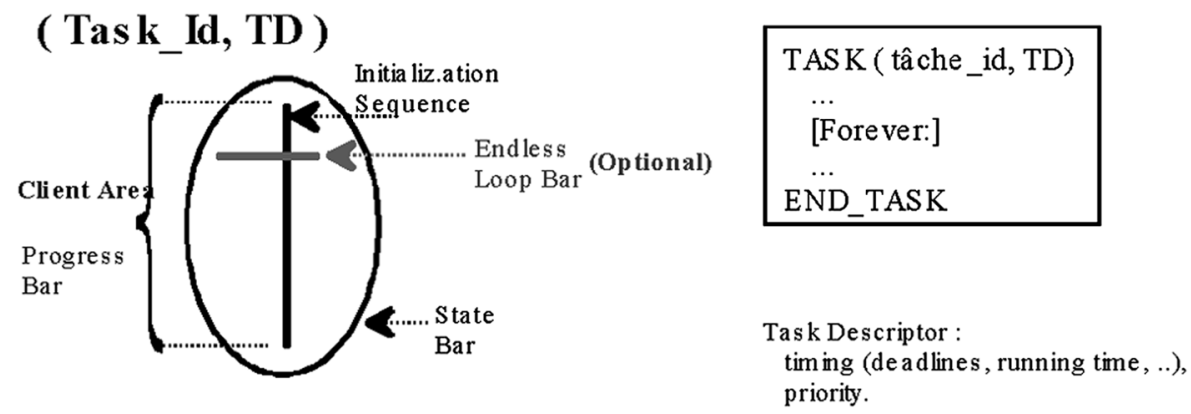

Fig. 3. Task Description.

LACATRE has two types of objects:

1. The programmable objects: the task, the interruption, the alarm, and the exception. These are the objects whose behaviour is user-defined.

2. The configurable objects: the semaphore, the mailbox, the message, the event, the resource, etc. These are the objects whose behaviour is completely defined by parameter values set up during creation.

\section{LACATRE Graphical symbols (Objects):}

The graphical symbol of a LACATRE object is made of basic elements (lines) called areas from which start and end linking symbols of LACATRE connections (system calls). There are three types of areas:

1. State area: where LACATRE state system calls (creation) are anchored and which may be applied to all LACATRE objects;

2. Progress area: specific to programmable objects. It is an oriented line that describes the sequence of LACATRE system calls. It may be altered by means of LACATRE algorithmic forms (IF, WHILE,);

3. Action area: attached to a configurable object and has a specific name according to the concerned object.

\section{Connections:}

The LACATRE system calls are the ones used by the designer to express the interactions among objects. They are classified in 3 categories:

1. State system calls: allow for the modification of the state of an object: creation, deletion,
2. Action system calls: generate a communication/synchronization relation between programmable objects (mainly tasks) with the help of configurable objects.

3. Progress system calls: provide straight synchronization between programmable objects.

After the task design is finished, the building blocks of the textual representation thereof are assigned maximum execution times and input as task models into the program architecture of the Specification PEARL system model as TSTDs (Timed State Transition diagrams, [13], see Section 6). The trigger conditions are converted into start states, whereas the building blocks of the tasks are assigned working states. End states are produced for end-of-task, error and timeout conditions. The tasks in TSTD form are used later on in the integration verification.

\subsection{Program Model Verification}

The program model in LACATRE is translated to Shaw's CRSM (Communicating Real-time States Machines) internal representation in order to perform the analysis of the designed tasks [15]. This enables the verification of:

- structure (well formed tasks perform smoother and safer and at the same time the basic building blocks are identified, which can be assigned maximum execution times to perform timing analysis) and

- behaviour (possible deadlocks, bottlenecks, etc. can be detected and avoided by the execution of the CRSM program model). 


\section{Implementation and Analysis: ObjectPEARL}

For the implementation of the application, objectoriented programming language ObjectPEARL is devised $[37,38]$. Like Specification PEARL, it is based on programming language PEARL that was developed in late 60 -ies by engineers from different real-time system domains. It has been refined during the years and its latest version (PEARL90) includes several elements of modern programming languages, but is not object-oriented. It explicitly supports tasks and tasking operations, semaphores, signals, etc. However, for the consistent use in hard real-time systems, some restrictions must be applied.

To allow for straightforward translation from object-oriented design tools and methodologies, traditional elements of real time systems are represented by means of object-oriented notation. Thus, all elements of LACATRE and Specification PEARL notation can be translated into ObjectPEARL's code.

\subsection{Object-oriented Extension of PEARL}

For introduction of classes, similar approach as in $\mathrm{C}++$ was used. In both cases, a class is defined as an extension to the structure. Because of simplicity of code generation and execution time analysis, it was decided not to use multiple inheritances of classes. Therefore, a class can be descendent of only one parent class. All traditional elements of classes were included (i.e. variables, methods, constructors and destructors). Like other object-oriented languages, ObjectPEARL also supports polymorphism through virtual methods.

In addition, a special type of variables called virtual variables or properties was introduced. Properties behave as ordinary variables with two additional methods. The first method is used for reading the variable and the second is used to change its value. Properties operate in similar manner as those used in the COM automation model [29].

There are also special kinds of properties that are declared only for specification purposes and no code is associated with them. They can represent certain non-program-specific attributes.
For example, attributes that represent parameters of graphical appearance of an object in LACATRE or non-programmatic attributes of Specification PEARL can be given in this way. This allows two-way translation between textual and graphical representation of the application. As an ultimate goal, no additional editor or tool for coding should be necessary to develop an application.

\subsection{Object-oriented Representation of Tra- ditional Elements of Real-Time Appli- cations}

To exploit the advantages of object-oriented approach, traditional elements of real-time systems are also represented as classes and objects. To provide for this, a way to represent tasks, semaphores, bolts, signals etc. was studied.

Tasks in PEARL and some other traditional programming languages are considered as a special kind of procedures, which can be executed in parallel under control of the operating system. For this purpose, a set of well-defined tasking operations is defined.

On the other side, objects are traditionally considered as extensions to the static data types. Objects do not have direct control over their execution and there is usually no provision for tasking.

Both views can be combined if task is considered as an object with a method that represents the main task behaviour and with several methods for tasking. Similar approach is used in Java.

General task in ObjectPEARL is declared as shown in Figure 4.

Data types like TASKCONTEXT and TASKSTATE are system-defined classes that represent context of the task, its current state, etc. Variable FContext and methods SaveContex and RestoreContex are declared as private components of the class and are used only within the operating system. A set of Get and Set methods are declared in a similar way and are used to access an internal state of the task and to perform low level tasking operations. MainProc is the main task 


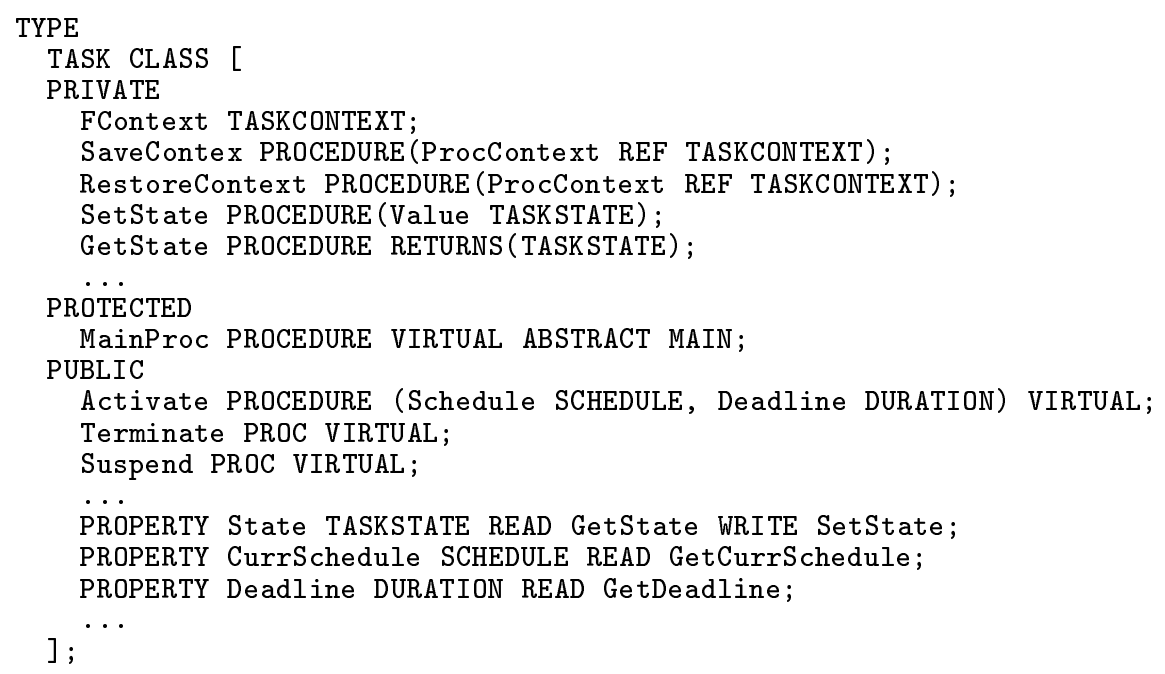

Fig. 4. Declaration of a Task in ObjectPEARL.

execution method. Tasking operations are declared as public to allow the access from outside of the object.

Similar to this, other elements of real time systems can be represented as classes. For example, a semaphore can be declared as in Figure 5.

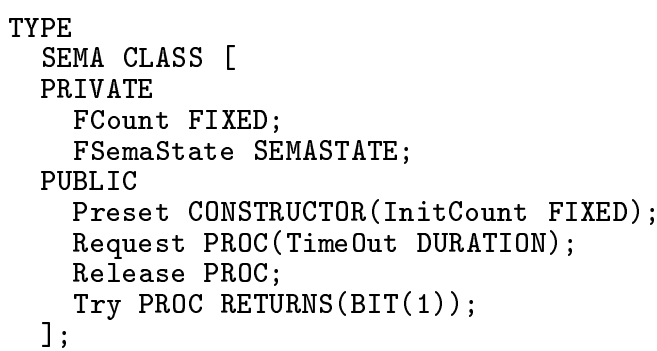

Fig. 5. Declaration of a Semaphore.

Like in tasks, private variables FCount and FSemaState are used for internal implementation of a semaphore. Again, private variables and implementation of methods is under direct control of the operating system. Because of the need for temporal predictability of the programs in ObjectPEARL, there is an additional parameter associated with Request method that serves as deadline on waiting for the semaphore to be released.

Benefits of object-oriented description can be fully utilised when there is already a wide set of predefined data types (classes) and when components are divided into specialised groups (e.g. periodic and a-periodic tasks).

\subsection{Compiler for ObjectPEARL With Inte- grated Execution Time Analyser}

Parallel to the programming language design, a compiler was implemented. The goal was to develop a modular and flexible compiler that could be easily modified based on results of theoretical research of the development environment. Compiler should be also target-system independent although in its current version it generates code only for one processor. Nevertheless, compiler should generate efficient code, thus several optimization techniques were included into the compiler.

One of the most important issues in implementation of the compiler/analyser was realistic estimation of worst-case execution times of tasks, which is the basis for timing analysis on higher levels. Too pessimistic execution times of tasks could declare a system unfeasible and require unnecessary effort to improve it. On the other hand, too optimistic estimations cause deadline misses when an application is executed on a real system. Worst-case execution time analysis can also be done on the specifications or on the program code but most accurate results can be achieved by execution analysis of compiled code or by direct measurement of the program on the target.

Compiler for ObjectPEARL was implemented with Delphi development environment and runs on Windows 9x or Windows NT platform. It 
uses simple recursive descent parsing algorithm with semantic routines for code generation and temporal analysis. In its current version, it generates code for Motorola's ColdFire microcontroller.

Translation of ObjectPEARL code into the standard PEARL was also studied but there were several semantic discrepancies between both languages (e.g. visibility of the class members). These differences cannot be easily considered by using simple translation techniques.

\subsection{Translation Process and Execution Time Analysis}

Standard PEARL programs are divided into two parts: system and problem part. In the system part hardware specific peculiarities are defined, e.g. input/output devices, communication devices etc. System part can be generated directly from the Specification PEARL. There are other attributes in Specification PEARL that are not directly used in code generation but they can be used to improve validation process of the application. For example, in Specification PEARL there is a specification of available memory for the program code. Compiler can easily check if the compiled code is larger than the specified storage room. Other attributes can be used during execution time analysis (e.g. processor speed, memory wait states, etc.).

In the problem part algorithmic elements of the application are presented independently of the hardware. Problem part can be directly generated from LACATRE notation. By using object representation of all real time elements, this translation can be simple and straightforward.

Worst-case execution time analysis is performed in two phases. In the first phase, structure of the program by means of blocks, loops, decisions, etc., is identified. Then for all linear parts of the code maximal execution time is estimated. Similar to the code generation, execution time analysis is highly dependent on the microprocessor used. Because of this, basic execution time analysis should be integrated within a lowlevel code generator. Thus, in ObjectPEARL compiler each object for code generation also has a corresponding method for execution time estimation. High-level temporal analysis is per- formed on the structure of the program using simple recursive rules [36].

Because of different mechanisms of modern microprocessors to improve overall performances (i.e. cache, pipeline, etc.), the estimated execution times based only on compiled code can be too optimistic. Execution times available for individual instructions are given for the ideal situation where instruction is already in the cache and no stall in pipeline or collision on address and data busses are considered. Although we tried to improve algorithm for execution time calculations, result was not always acceptable.

For that reason it was decided to implement direct measurement of execution times of sequential parts of code on the target processor. In this sense, the debug module of the target processor was utilized, featuring background run-time debug support. With this module, it is possible (over a separate external bus) to stop or run a program on the processor, read or write into the registers and memory, set breakpoints, etc. This allows to load the compiled code into the processor's memory, run segments of code and measure the elapsed time.

The results of the execution time analysis are estimated execution times of tasks. These results with some additional information (e.g. deadlines of tasks, activation conditions, etc.) provide the data for further schedulability analysis.

Idiosyncrasy of the microprocessors is a fundamental impediment in accurate execution time estimation. Because of that, generality of the approach is limited: the timing analysis has to be adapted for every new processor. However, by becoming widely used, BDM to some extend standardises the measurement of execution times at least within the Motorola family of microprocessors.

\section{Verification of the Integral Design by Co- Simulation}

According to [25], for most applications running in real-time their computational model can be expressed in the form of the following "equation":

Real-time program model $=$ Data flow model + State automaton + Timing limitations 
The TASKs of the system represent the basic program units whose main properties are their trigger conditions and timing limitations as well as being part of a MODULE and COLLECTION. This information is sufficient to build a rough program model, but it is not enough to determine the feasibility of the program configuration on the model of the target system. Therefore, they have been extended by timed state transition diagrams, which represent the dynamic behaviour of tasks, whereas their execution can be simulated on the model of the target system.

Timed State Transition Diagrams (TSTD) act as timed finite state automata and consist of:

- start states (task trigger conditions),

- transient states (working states) and

- final states (finalisation actions).

The connections between states represent the applications' progress in time. All connections are local (i.e.: bound to the states of one task). Inter-task co-operation is modelled by the state actions-system calls of the operating system. These also enable the fulfilment and checking of continuation preconditions of states. The operating system is invisible to the user except for its system calls and configuration, which is setup by setting the parameters of the KERNEL station.

Every state contains the following data:

- state type (start, working or final state),

- precondition for the states' execution (trigger condition for a start state),

- timeout condition (shortest and maximum execution times),

- timeout action (the state where the execution is diverted in case the timeout occurs),

- connection to the next state in case the execution continues successfully,

- activities, which are carried out within the execution of this state (PEARL-comment or system call).

System calls, which trigger specific task states, make resource requests and change the state of the system. Interfaces and ports are referenced in communication system calls as configured in the hardware/software architecture.
The activities within a state are a set of actions, which are carried out while the task is in the state. It is foreseen that the actions may be either a system call and/or a mini-specification (PEARL comment). Their execution time is estimated by the designer and used while setting the time-out interval for the state.

\section{Verification Method}

After the coherence of the design has been checked, the feasibility of the modelled system is validated. It has been established that co-simulation of the execution of the tasks on the specified (hardware and software) system architecture is the most appropriate verification method for our methodology.

The program as well as hardware models may be modified if necessary due to the feasibility estimation from the co- simulation or subsequent execution time analysis that may differ from the execution time estimates in the program model.

The method of the next critical moment cosimulation of execution with time boundaries has been chosen as the method for verification of the designs. The boundary time values are considered along with the variables' values, as it is usual in the program verification practice. For each state the shortest and the longest transaction times are checked.

By taking the shortest and the longest transaction times through the task states of the system, the time domain is sufficiently covered to be able to generalise the results of arbitrary transaction time of every state and herewith also the system as a whole.

The following concept of correctness for the described method of verification has been defined: "The system fails in the case when during co-simulation the system reaches an undefined state or its predefined time frame is violated and no timeout-action is defined."

The station clock rate is translated into the step size of the station in the co-simulation and is used when the next event time is being calculated. The station components are not relevant for the co-simulation, but they may be subject to compatibility checks and their time parameters must be used later on in the schedulability analysis. 


\section{Conclusion}

The pragmatic combination of the tools presented in this paper enables parallel design of the hardware and the software parts and offers textual as well as graphical notations for both domains. It allows for early mapping of the problems onto the appropriate hardware or software solutions and their design. Further, early verification and validation of functional and timing properties is enabled considering behaviour of hardware and software components of the application.

By taking already existing tools, problems were anticipated from the beginning. The main weak points of the tools in the combined approach include:

- lack of the means for requirements engineering of the system,

- lack of the possibility of direct mutual impact of the solutions between LACATRE and SPEARL,

- PEARL does not explicitly support certain useful LACATRE constructs (e.g. mailboxes),

- in the current version of LACATRE it is not possible to change the graphical model by modifying the textual form of the application,

- LACATRE does not allow for hierarchical top-down design of larger applications, it does not itself explicitly deal with temporal properties and does not support deadlinedriven scheduling.

Most of the listed problems could be solved by applying certain changes in the forthcoming versions of LACATRE, SPEARL and OPEARL. Apart of that, for the successful use of the methodology an integrating environment should be developed.

\section{References}

[1] G. AgHA, The Structure and Semantics of Actor Languages, in J.W. de Bakker, W.P. de Roever, and G. Rozenberg, editors, Foundations of ObjectOriented Languages, pp 1-59, Springer Verlag, 1991.
[2] F. Balarin, M. Chiodo, P. Giusto, H. Hsieh, A. Jurecska, L. Lavagno, C. Passerone, A. SANGIOVANNI-VinCENTElli, E. SENTOVICH, K. SUZUKI, B. TABARRA, Hardware-Software CoDesign of Embedded Systems: The POLIS Approach, Kluwer Academic Publishers, 1997.

[3] G. Booch, I. Jacobson, J. Rumbaugh ET. AL., The Unified Modeling Language for Object-Oriented Development Version 1.0, UML Notation Guide, UML Summary, UML Semantics; Rational Software Corporation, January 1997 and the UML 1.1 update of Sept. 1997.

[4] G. Borriello, P. Chou, Ross B. Ortega, Embedded System Co-Design: Towards Portability and Rapid Integration, Hardware/Software Co-Design, pages 243-264, Kluwer Academic Publishers, 1996.

[5] A. Burns, A. J. Wellings, HRT-HOOD: A Structured Design Method for HRTS, Real-Time Systems, Vol. 6, pp. 74-114,1994.

[6] Z. Chaochen, Ji Wang, A. P. Rawn, A Formal Description of Hybrid Systems, Hybrid Systems III, eds. R. Alur and T. Henzinger \& E. Sontag, LNCS 1066, Springer-Verlag, 1996.

[7] M. Colnarič, D. Verber, W. A. Halang, A Real-Time Programming Language as a Means of Expressing Specifications, Proceedings of 21 st IFAC/IFIP Workshop on Real-Time Programming (WRTP'96), Gramado, RS, Brazil, November 1996.

[8] M. Colnarič, D. Verber, R. GumzeJ, W. A. HaLANG, Implementation of Real-Time Embedded Control Systems, Real-Time Systems, Kluwer Academic Publishers, May 1998.

[9] C. DIETZ, Action Diagrams, Proceedings of 22nd IFAC/IFIP Workshop on Real-Time Programming (WRTP'97) (Preprints), September 15-17, 1997, Lyon, France.

[10] T. J. ERIKSEn, S. T. HeIlmanN, M. HoldgaARd, A. P. RAVN, Hybrid Systems: A Real-Time Interface to Control Engineering, Proceedings of 8th Euromicro Workshop on Real-Time Systems, IEEE, pp. 114-120, 1996.

[11] R. Gumzej, M. Colnarič, J. P. Babau, J. SkUBICH, Hardware Architecture Components for RealTime Systems Design, in Proceedings of the 7th Electrotechnical and Computer Science Conference ERK'98, Vol. A pp. 41-44, Portoroz, Slovenia, September 1998.

[12] R. Gumzej, M. Colnarič, D. Verber and W. A. HALANG, Towards standard-based specification and design of embedded real-time systems, in Proceedings of Euromicro'98, Väasterăs, Sweden, August 25-27, 1998.

[13] R. GuMZEJ, M. COLNARIČ, An approach to real-time systems co-design and verificaiton, in Proceedings of the IASTED international conference on Control and Applications, Cancun, Mexico, May 2000.

[14] R. GUPTA, Co-Synthesis of Hardware and Software for Digital Embedded Systems, Kluwer Academic Publishers, 1995. 
[15] Z. HuAnG, A. Legait, M. MaranZana, E. NIEL, J. J. SChWARZ, J. SKUBICH, Techniques for the Behaviour Verification of Real-Time Multitasking Components, 14th IFAC World Congress, 5-9 July 1999, pp. 6, Beijing, Chine.

[16] International standard ISO/IEC 9126: Information technology - Software product evaluation - Quality characteristics and guidelines for their use, First edition 1991-12-15, Reference number ISO 9126 : 1991.

[17] International standard ISO/IEC 9127: Information processing systems - User documentation and cover information for consumer software packages, First edition 1988-09-01, Reference number ISO 9127 : $1988(\mathrm{E})$.

[18] E. Kligerman, A. D. Stoyenko, Real-Time Euclid: A Language for Reliable Real-Time Systems, IEEE Transactions on Software Engineering, Vol. 12, No. 9, September 1986, pp. 941-949.

[19] I. LeE, S. DAVIDSON, R. GERBER, Communicating Shared Resources: A Paradigm for Integrating Real-Time Specification and Implementation, Foundations of Real-Time Computing: Formal Specifications and Methods, Kluwer Academic Publishers, 1991.

[20] A. LyONS, UML for Real-Time Overview, ObjecTime Limited, April 1998.

[21] Full PEARL, DIN 66253, Part 2.

[22] Distributed system PEARL, DIN 66253, Part 3.

[23] H. De Man, I. Bolsens, B. Lin, K. VAN Rompaey, S. VERCAUTEREN \& D. VERKEST, Co-Design of DSP Systems, Hardware/Software Co-Design, pages 75-104, Kluwer Academic Publishers, 1996.

[24] V. J. MoOnEY III, Hardware/Software Co-Design of Run-Time Sytems, School of Electrical and Computer Engineering, Georgia, PhD thesis, Sep. 1998.

[25] A. K. MoK, Towards Mechanization of Real-Time System Design, Foundations of Real-Time Computing: Formal Specifications and Methods, Kluwer Academic Publishers, 1991.

[26] L. MOTUS, T. NAKS, Formal timing analysis of OMT designs using LIMITS, Computer System Science and Engineering, Vol. 13, No. 3, pp. 161-170, 1998.

[27] ObjecTime Limited, ObjecTime Overview, 1994.

[28] J. S. OstrofF, A Visual Toolset for the Design Of Real-Time Discrete Event Systems, IEEE Transactions On Control Systems Technology, May 1997.

[29] D. Rogerson, Inside COM, Microsoft Press, 1997.

[30] J. Rumbaugh, B. SElic, Using UML for Modeling Complex Real-Time Systems, Rational Software Corporation, ObjecTime Limited, March 1998.

[31] J. J. SchWARZ, J. J. SKUBICH, Graphical programming for Real-Time Systems, Control Engineering Practice, Vol. 1, No. 1, pp. 43-49, 1993.
[32] A. C. SHAW, Communicating real-time state machines, IEEE Trans. Software Engineering, Vol. 18, No. 9, pp. 805-816.

[33] S. SHLAER, S. MELLOR, Object-oriented systems analysis: modelling the world in data, Prentice Hall, Englewood Cliffs, NF, 1998.

[34] Traore I., Sahraoui Abd-El-Kader, A Multiformalism Specification Framework with Statecharts and VDM, Proceedings of 22nd IFAC/IFIP Workshop on Real-Time Programming (WRTP'97) (Preprints), September 15-17, 1997. Lyon, France.

[35] OMG Unified Modeling Language Specification (draft), Version 1.3, March 1999.

[36] D. Verber, Programming and timing analysis of hard real-time systems, Master thesis, 1997, University of Maribor

[37] D. VERBER, Object Orientation in Hard Real-Time System Development, Doctoral thesis, 1999, University of Maribor, Slovenia.

[38] D. Verber, M. COLNARIČ, Object Oriented Extension to Development of Hard Real-Time Systems, to appear on SCI/ISAS 2000, Orlando, Florida.

Received: April, 2000 Revised: June, 2000 Accepted: July, 2000

Contact address:
Roman Gumzej
University of Maribor,
Faculty of Electrical Engineering Computer science,
Smetanova 17
2000 Maribor
Slovenia
e-mail: roman. gumzej@uni-mb.si
Jean-Philippe Babau
INSA de Lyon+
Bat 502-L3i
20 Av A. Einstein
69621 Lyon, France
e-mail: jpbabau@if. insa-lyon.fr

ROMAN GUMZEJ joined the Faculty of Electrical Engineering and Computer Science at the University of Maribor, Slovenia, in 1994, as a junior research follow. He received his Master of Science and Doctor of Science degrees in 1997 and 1999 respectively. He is currently a teaching assistant and research engineer at the same faculty.

His main research interests are real-time systems, their co-design, operating systems as well as verification and validation. He is a member of the IEEE Computer Society.

DOMEN VERBER is assistant professor at the Faculty of Electrical Engineering and Computer Science, University of Maribor, Slovenia. He received his Master of Science and Doctor of Science degrees in 1996 and 1999 respectively.

His main research interests are real-time programming languages, object orientation, temporal and schedulability analysis of task, and application design techniques and methodologies. He is a member of the IEEE Computer Society. 
MATJAŽ COLNARIČ is associate professor at the Faculty of Electrical Engineering and Computer Science, University of Maribor, Slovenia, from which he also received his Master of Science and Doctor of Science degrees in 1983 and 1992, respectively. He chairs the Real-Time Systems Laboratory and teaches courses on microprocessors, real-time systems, and algorithms and data structures.

His main research interests are related to embedded real-time control systems, their hardware and system architectures, operating systems, programming languages as well as application design techniques and methodologies therefor.

Dr. Colnarič has authored, or co-authored, some 80 journal and conference papers and book chapters, mainly in the real-time area. He served in programming committees of a number of international conferences, he organised special sessions and chaired them. He is a member of the IEEE Computer Society and its TCs on Real-Time Systems and Complexity in Computing. He is also a member of IFAC Technical Committee on Real-Time Software Engineering.

JEAN-PHILIPPE BABAU is an assistant professor in the department of computer science at the INSA (engineer school) of Lyon. He received his $\mathrm{PhD}$ in computer science from the University of Poitiers in 1996. His research interests include the design, object-oriented techniques, and the analysis of temporal behavior for complex real-time systems.

JACQUES SKUBICH is an assistant professor at the Department of Computer Engineering \& Inf. Technology at INSA de Lyon, France from which he received his Ph.D. in Applied Computer Science and Automation in 1983. His main lecturing activities are related to Operating Systems, Software Engineering \& Real-Time Systems, in particular by means of graphical programming. He is a member of programming committees of a number of Conferences and has been the general Chairman of WRTP'97 in 1997. He is a member of the IF AC Technical Committee on Real-Time Software Engineering. 